\title{
Estructuras del sentir en contextos de politicidad juvenil ${ }^{*}$ Structures of feeling in contexts of youthful politicity
}

\author{
Sandra Poliszuk ${ }^{* *}$
}

\section{Resumen}

El presente trabajo se propone analizar las prácticas comunicacionales en las redes sociales digitales de jóvenes que integran dos agregaciones estudiantiles de la ciudad de Viedma, Río Negro, a fin de comprender el lugar significativo que tienen dichas prácticas en las estructuras del sentir, presentes y activas en contextos y procesos actuales de politización juvenil.

La estructura del sentir es una propuesta conceptual planteada por Raymond Williams -inscripta en la tradición de los Estudios culturales ingleses- a modo de hipótesis cultural como un intento por comprender los emergentes sociales que se expresan en nuevas formas de sentir y pensar frente a los sentimientos ya "precipitados" o articulados de modo pleno en las instituciones de un período determinado (Williams, 1997).

El trabajo recupera la trayectoria grupal de dos agregaciones juveniles: una agrupación estudiantil universitaria y un grupo de estudiantes secundarios de una radio escolar comunitaria. Los cambios en la estructura del sentir se rastrean en la "conciencia práctica" de estas dos agregaciones.

\footnotetext{
* Artículo recibido 30 de julio de 2016. Aceptado 21 de febrero de 2017.

** Magister en Ciencia Política y Licenciada en Comunicación Social. Docente - investigadora en la Universidad Nacional del Comahue. Correo electrónico: spoliszuk@yahoo.com
} 


\section{Abstract}

This paper intends to analyze the communicational practices in digital social networks of young people who integrates two student aggregations in the city of Viedma, Río Negro, in order to understand the significant place that such practices have in the structures of feeling, presents and actives in current contexts and in the processes of youth politicization.

The structure of feeling is a conceptual proposal poised by Raymond Williams- registered in the tradition of the English cultural studies- by way of cultural hypothesis as an attempt to understand the emerging socials which expresses in new ways of thinking and feeling in front of feelings "already precipitated" or "fully articulated manner" in the institutions of a certain period (William, 1997).

This paper recovers the group trajectory of two juvenile aggregations: a university student group and a group of secondary school students in a community radio. Changes in the structure of feeling are tracked in the -practical consciousness- of these two aggregations.

\section{Palabras clave}

Prácticas comunicacionales- estructuras del sentir- identidad narrativa.

\section{Keywords}

Communicational practices-structure of feeling-narrative identity.

\section{Introducción}

La irrupción de las redes sociales digitales ha generado numerosos debates, reflexiones y estudios sobre su impacto en los procesos comunicacionales, sus alcances y sentidos en el universo práctico y simbólico de diversos grupos socioculturales.

Como nuevo escenario de interacciones, Internet y las redes sociales digitales conllevan transformaciones globales en las formas comunicacionales, sin embargo, sus usos, formas de socialización y significaciones albergan apropiaciones diversas y generan entramados socioculturales diferentes. Esta es una de las premisas que guían el presente trabajo cuyo objetivo es analizar las prácticas comunicacionales en las redes sociales digitales de jóvenes que integran dos agregaciones estudiantiles de la ciudad de Viedma, Río Negro, y su impacto en las estructuras del sentir desde una doble mirada: por un lado, se busca comprender algunos procesos comunicacionales más amplios que conllevan la irrupción de las mencionadas redes y forman parte del 
horizonte cultural de una época; por otro, se indaga en las significaciones y sensibilidades que generan dichas prácticas entre jóvenes que participan en contextos y procesos actuales de politización juvenil.

La estructura del sentir es una propuesta conceptual planteada por Raymond Williams -inscripta en la tradición de los Estudios culturales ingleses- a modo de hipótesis cultural como un intento por comprender los cambios cualitativos en las formaciones, instituciones y creencias, sus conexiones y elementos en una generación o un período determinado (Williams, 1997). La productividad de esta hipótesis radica, entre otros aspectos, en la posibilidad de analizar procesos y elementos configuradores del presente, activos o "en solución"; para Williams, son las formaciones emergentes con las que se relacionan las estructuras del sentir (Williams, 1997).

Este análisis forma parte de los resultados de una investigación que se desarrolló entre 2011 y 2014 en Viedma, ciudad situada en la región norpatagónica de la República Argentina. El proyecto se acreditó institucionalmente en la Universidad Nacional del Comahue ${ }^{1}$. La investigación se vio enriquecida a partir de un plan conjunto con el Cuerpo Académico Comunicación, Cultura y Desarrollo del Proyecto Culturas Juveniles de Mexicali, de la Universidad Autónoma de Baja California, con la firma de un convenio específico de intercambio entre las dos universidades.

El trabajo recupera la trayectoria grupal de dos agregaciones juveniles: una agrupación estudiantil universitaria y un grupo de estudiantes secundarios de una radio escolar comunitaria, y analiza las formas de sentirse de sus integrantes en las redes sociales digitales cuando estas se vinculan con sus vivencias de militancia cotidiana.

Nuestra hipótesis es que la experiencia de tiempo-espacio y la dimensión comunicativa de la intimidad que se vivencia en las redes sociales digitales ${ }^{2}$ impactan, crecientemente, en las formas de sentir la militancia estudiantil. Las sensibilidades que se construyen en las redes sociales digitales, vinculadas con formas concretas de expresar los sentires, van naturalizándose y configurando un particular modo de vivir la militancia en cada agregación estudiada.

\footnotetext{
1 El proyecto de investigación titulado Los jóvenes y las redes sociales virtuales como espacios resignificadores de culturas políticas (04/V070) se desarrolló en el Centro Universitario Regional Zona Atlántica (CURZA), de la Universidad Nacional del Comahue. También se recuperan en este trabajo los aportes de una investigación en proceso, acreditada en la Universidad Nacional del Comahue, e iniciada en el año 2015 en el CURZA. El proyecto se titula Políticas de visibilidad y agendas de lo juvenil en Viedma (04/V095).

2 Partimos comprendiendo a las redes sociales digitales como medios interactivos que se asientan sobre una lógica reticular, diferente a la lógica del broadcasting, y se distinguen por la colaboración activa de los usuarios (López y Ciufoli, 2012). Una red social virtual paradigmática de esta lógica es Facebook, aunque no es la única ni la más antigua.
} 


\section{Puntos de partida}

\subsection{Comunicación en la cultura}

Este trabajo parte de comprender la comunicación en la cultura, es decir, en el marco de contextos socioculturales y políticos cambiantes. Se recuperan los aportes de la propuesta teórica de Jesús Martín Barbero sobre las mediaciones, quien aborda los procesos culturales "no desde los medios, sino desde las mediaciones, desde las articulaciones entre prácticas de comunicación y movimientos sociales y las diferentes temporalidades y la pluralidad de matrices culturales" (Martín-Barbero, 1987: 81).

Martín Barbero (1999: 21) señala:

De lo que se trata [...] es de indagar lo que la comunicación tiene de intercambio e interacción entre sujetos socialmente construidos, y ubicados en condiciones que son, de parte y parte aunque asimétricamente, producidos y de producción, y por tanto espacio de poder, objeto de disputas, remodelaciones y luchas por la hegemonía.

En relación con la concepción de hegemonía, afirma Martín Barbero (1987: 72):

La dominación, lejos de ser un proceso de imposición desde el "exterior" de lo social y de la cultura, es un proceso en el que una clase se hace hegemónica cuando logra representar intereses diferentes de las clases populares y, además, en la medida en que los sectores populares se reconocen "adentro" del proceso hegemónico, lo asumen, lo interiorizan, son conformistas con él. En ese proceso, se transforman permanentemente las culturas y las identidades dominantes y populares.

También el concepto de mediatización de la cultura adquiere importancia en el estudio de los procesos actuales de producción social de los sentidos y construcción identitaria. Por mediatización se hará referencia en este trabajo a las transformaciones que se están dando en los procesos de producción de sentidos vinculados a la presencia de medios y dispositivos tecnológicos que forman parte de las dinámicas sociales y culturales más amplias ${ }^{3}$. La mediatización de la cultura, desde esta perspectiva, se relaciona con las transformaciones en los modos de producir, distribuir y poner en circulación los bienes simbólicos (Thompson, 1998).

En este marco, asumimos la caracterización de Hine (2004) de Internet como cultura y como artefacto cultural. Por un lado, Internet representa un lugar donde se gesta una cultura: el ciberespacio aparece como escenario de interacciones relevantes. Por otro,

\footnotetext{
${ }^{3}$ Desde este enfoque comprendemos que los dispositivos tecnológicos no son instrumentos o canales neutrales y externos a las prácticas sociales, cuyo principal objetivo sea puramente la transmisión de información. Un dispositivo tecnológico se imbrica con las prácticas y los procesos sociales que dinamizan sentidos, rutinas y modalidades de apropiación. Según Eliseo Verón (1997), "tecnología de comunicación" y "medio de comunicación" son conceptos que designan cosas diferentes. Un medio comporta la articulación de una tecnología de comunicación a modalidades específicas de utilización (en producción y en recepción). La distinción asume que no hay determinismo tecnológico respecto de los usos sociales. Una nueva tecnología de comunicación no determina, lineal y mecánicamente, prácticas sociales específicas de producción y de consumo, aunque el discurso tecnocrático que acompaña la difusión de nuevos dispositivos pueda estar tentado de alimentar esa ilusión. En consecuencia, un mismo dispositivo tecnológico puede insertarse en contextos de utilización múltiples y diversificados.
} 
Internet es comprendido como un artefacto cultural que está en todas partes, pero no del mismo modo, artefacto no neutral que genera diferentes entramados sociales según su uso. Al respecto, sostiene Hine (2004: 42): "decir que Internet es un objeto o artefacto cultural como cualquier otro, no implica que sea el mismo objeto para todas las personas".

\subsection{Identidad narrativa}

La noción de identidad narrativa de Paul Ricoeur ${ }^{4}$ es una categoría de la práctica; es la narración que hace una persona de sí misma y para sí misma. Para este autor, las historias se narran, pero también se viven en el modo de lo imaginario. Una vida no es sino un fenómeno biológico hasta tanto no sea interpretada. $Y$ en la interpretación, la ficción desempeña un papel mediador considerable (Néspolo, 2007). Desde este enfoque, no hay identidad por fuera de la representación de la narrativización, es decir, de la ficción. En el despliegue de lo ficcional, el sujeto se conoce y reconoce en una trama operando en ella la multiplicidad de las identificaciones que modelan su experiencia. Pero es en el marco de algún orden cultural donde esa trama se vuelve realidad, es decir, cobra o no sentido y autenticidad. Facebook, la red más usada entre los jóvenes estudiados, centraliza en una única identidad las múltiples voces, produciendo un efecto de transparencia y autenticidad al trasladar las identidades offline a la red en un poderoso intento de hacer coincidir un nombre propio con una vida real (López y Ciuffoli, 2012). De este modo, se refuerza la idea de un yo que se auto-construye a sí mismo mediante narraciones con enfáticos "efectos de real", cuya veracidad no requiere ser comprobada o validada por alguna fuente o autoridad para configurarse como un mundo posible porque es autorreferencial. Esto nos lleva a pensar que las construcciones identitarias en las redes sociales digitales presentan un fuerte componente ficcional. Las narrativas vivenciales que se despliegan en dichas redes son uno de los modos de las "escrituras del yo" que ingresan al espacio biográfico ${ }^{5}$ en tanto horizonte interpretativo desde el cual es posible lograr una lectura

\footnotetext{
4 Frente a las "filosofías del cógito" que plantean un núcleo no cambiante de la personalidad, Ricoeur propone el concepto de sí mismo como otro, aunando las nociones de mismidad e ipseidad en un mismo centro al que sólo accede el sujeto por medio de un rodeo narrativo dado en llamar "hermenéutica del sí" (Néspolo, 2007). En este trabajo se retomarán las lecturas de Paul Ricoeur que proponen Leonor Arfuch (2007) y Néspolo (2007).

${ }^{5}$ El Espacio Biográfico, según Leonor Arfuch (2007), configura un horizonte interpretativo capaz de dar cuenta del énfasis biográfico que caracteriza un espacio/temporización en una simultaneidad de ocurrencias que pueden transformarse para lograr una lectura comprensiva en el marco de una lectura de época. Se trata así de una confluencia de múltiples formas, géneros y horizontes de expectativas donde circulan narrativas de vidas. De esta manera, el Espacio Biográfico, según Arfuch (2007:51), se compone de: "autobiografías, memorias, testimonios, historias de vida, diarios íntimos, secretos, correspondencias, cuadernos de notas, de viajes, borradores, recuerdos de infancia, autoficciones, novelas, filmes, video y teatro autobiográfico, entrevistas mediáticas, conversaciones, retratos, perfiles, anecdotarios, indiscreciones, confesiones, vidas variantes del show, la videopolítica, los relatos de vida de las ciencias sociales y la escritura académica".
} 
de época (Poliszuk, 2013). Es en dicho espacio donde confluyen actualmente múltiples formas, códigos y narrativas atravesadas crecientemente por el lenguaje multimedial y donde la subordinación de los géneros a la lógica de los formatos potencia la multiplicación infinita de los microrrelatos (Martín Barbero, 2002).

El devenir de una identidad está atravesado por códigos de inteligibilidad o patrones de representación a partir de los cuales se experimenta el mundo: la identidad, abierta a la experiencia del tiempo-espacio, es uno de ellos y será abordado en este trabajo.

Las narrativas inscriptas en redes sociales digitales plantean una particular construcción de la identidad del sujeto y esa particularidad obedece a un modo de experimentar el tiempo y espacio. Al referirse a la tecnocultura, Sergio Balardini (2006: 4) plantea un cambio en los parámetros de tiempo y espacio:

Producto del mundo de los chips y el procesamiento a velocidad de la luz, los tiempos se redujeron al instante, y, las distancias, de la mano al mouse. De allí que se diga que la información está a un click de distancia. En este sentido, la digitalización del mundo y la globalización de las comunicaciones y los mensajes, han generado una sensación de proximidad témporo-espacial que lleva el patio de la casa hasta el lugar más recóndito y al futuro como una forma del presente.

Hay una sensación recurrente vinculada con el parámetro témporo-espacial en las redes digitales que se expresa, entre los jóvenes estudiados, en una experiencia signada por la cercanía, la velocidad y la simultaneidad, una especie de presente continuo, en el cual todo sucede en tiempo real porque el intercambio es permanente.

Otro patrón de inteligibilidad que será abordado en este análisis es la delimitación de lo público y lo íntimo en los usos de las redes sociales digitales. Con la creciente aparición de lo íntimo en la escena contemporánea, adviene la dimensión comunicativa de la intimidad y ella se despliega en las redes sociales digitales desafiando aún más las delimitaciones nunca nítidas entre lo público y lo privado ${ }^{6}$ (Poliszuk, 2013).

\subsection{Estructuras del sentir}

La estructura del sentir o estructura de experiencia es una hipótesis conceptual que desarrolló Raymond Williams, en el marco de su perspectiva materialista cultural.

\footnotetext{
${ }^{6}$ Una indagación de las relaciones entre lo público y lo privado, sus delimitaciones canónicas, excede los alcances y objetivos de este trabajo. Asumimos algunos presupuestos básicos que presenta el enfoque no disociativo del binomio público/privado desarrollados en Arfuch (2007). En el marco de las reconfiguraciones e hibridaciones actuales entre lo público y lo privado, sus tensiones constitutivas, el análisis de las representaciones de la intimidad en las redes sociales virtuales nos aleja de las separaciones nítidas entre ambos espacios con límites e incumbencias "canónicas" específicas y nos acerca a un enfoque no disociativo de este binomio (Arfuch, 2007). Al recuperar los aportes de Elías y Chartier, Arfuch plantea el antagonismo entre la esfera íntima y la pública/social como el efecto de reglas, constricciones y dispositivos de poder que no han hecho sino incrementarse (incluidos los dispositivos actuales de autocontrol frente a la creciente exhibición de la intimidad), y en la disociación operada entre individuo y sociedad (Poliszuk, 2013).
} 
Esta hipótesis permite analizar procesos y elementos configuradores del presente, activos o "en solución". Williams busca captar los elementos emergentes de la vida social, "aunque a menudo en forma de perturbación o la modificación de las antiguas formas" (Williams, 1997: 157), que no pueden reducirse a los modos de pensamiento o de representación preexistentes. Define a la estructura del sentir o sentimiento como "una experiencia social que todavía se halla en proceso, que a menudo no es reconocida verdaderamente como social sino privada, idiosincrática e incluso aislante" y solo puede ser reconocida "en un estadio posterior" cuando en muchos casos se han convertido en "instituciones y formaciones" (1997: 155).

Su hipótesis es un intento por comprender los cambios cualitativos en las formaciones y creencias, sus conexiones y elementos en una generación o un período determinado (Williams, 1997).

Cáceres Riquelme y Herrera Pardo (2014: 188 y 190) definen la estructura del sentir o sentimiento como la "emergencia de una forma alternativa de experimentar (de sentir y de pensar) la totalidad social" y agregan: "lo que pretende develar la noción de Williams es precisamente de lo que todavía permanece como alternativo, emergente y aún no ha sido articulado sistemáticamente por el proceso social. Podemos decir, entonces, que es a partir de los intersticios, los puntos ciegos existentes entre un orden social dominante y sus alternativas es que se ubica la preocupación teórica de una noción como "estructuras de sentimiento".

Los cambios en las estructuras de sentimiento se rastrean en la "conciencia práctica", es decir, en los significados y valores tal como son vividos y sentidos activamente, algo que se palpa y nunca se atrapa del todo ${ }^{7}$. La conciencia práctica, para este autor, es lo que se está viviendo: "Del pensamiento tal como es sentido y del sentimiento tal como es pensado; una conciencia práctica de tipo presente dentro de una continuidad viviente e interrelacionada" (Williams, 1997: 155).

El término sentir acentúa una distinción respecto de los conceptos más formales de "concepción del mundo" o "ideología". Se enfoca en los significados y valores tal como son vividos y sentidos activamente, y las relaciones existentes entre ellos y las creencias sistemáticas o formales. Se trata de elementos afectivos de la conciencia y de las relaciones, que se vivencian corporal, anímicamente en un determinado momento histórico.

Las mutaciones y tensiones en las estructuras del sentir anticipan los cambios sociales que luego se consolidarán en las instituciones. Williams analiza las modificaciones y tensiones en las estructuras del sentir en distintos momentos de expresión de esos cambios. Se trata de procesos complejos y contradictorios que son comprendidos, además, en términos de luchas de clases y disputas de sentidos. Los mecanismos de

\footnotetext{
${ }^{7}$ Williams hace referencia a diferentes estructuras de sentimientos correspondientes a diferentes clases o formaciones emergentes. También señala que en un determinado período histórico pueden coexistir distintas estructuras de sentimientos relacionadas con el surgimiento o la fractura de una clase social.
} 
circulación cultural están atravesados y son la resultante de una determinada correlación de fuerzas en lucha. Los procesos de cambios en las estructuras del sentir le permiten a Williams explicar las formas evidentes de lo emergente y su relación con lo residual y lo dominante en el proceso cultural total.

En el marco de las transformaciones socioculturales producidas por el desarrollo del capitalismo, las experiencias de clase van adquiriendo nuevas configuraciones. El análisis de Williams reconoce en la utilización de mecanismos como la incorporación, la materialización de la desigualdad dentro de una cultura dominante, cuando los elementos de las clases subordinadas, que portan contenidos antagónicos, logran ser adaptados, procesados y fagocitados. Pero también el autor analiza los mecanismos que activan dimensiones anteriores del sentir, como así también las expresiones genuinamente creativas de lo nuevo (Boito, 2010).

\section{Marco metodológico}

La investigación que da sustento a este trabajo se enmarcó en una perspectiva cualitativa, fue de carácter generativo y se desarrolló en el marco de un diseño flexible, con una primera fase exploratoria y descriptiva, ya que no había antecedentes locales sobre el tema estudiado, para luego abordar la dimensión interpretativa del fenómeno, en términos de estudio de casos múltiples. Esta estrategia metodológica consiste en el "estudio intensivo de varios casos elegidos, no en virtud de su representatividad respecto de otros o de una generalidad, sino, por el contrario, en función de sus particularidades, a partir de su originalidad" (Borobia, 2015: 233).

Trabajamos sobre tres dimensiones o niveles de análisis: a) estructural, que consistió en relevar los espacios de interacción y encuentro de los jóvenes y los equipamientos tecnológicos digitales utilizados; b) situacional, centrado en estudiar el contexto sociocultural y comunicacional en el que se desenvuelven los jóvenes; y c) simbólico, consistente en analizar la producción de sentidos que posibilitan a los jóvenes estar juntos y el significado que adquiere la tecnología ${ }^{8}$ y los medios de comunicación en esos procesos.

La población para este estudio fueron los jóvenes de Viedma, provincia de Río Negro, que integren diversas agregaciones juveniles y transiten diferentes etapas de la educación universitaria y en los últimos años de la educación media.

Según la perspectiva general de esta investigación, la recogida y el análisis de datos operó de manera simultánea, a medida que fue avanzando el proceso.

\footnotetext{
${ }^{8}$ Desde la perspectiva planteada en la investigación, las tecnologías son estudiadas teniendo en cuenta sus usos sociales, entendidos estos no solo en su utilización, sino principalmente en los sentidos que acerca de ellos producen los jóvenes. Es decir, la tecnología se define por lo que los jóvenes y las agregaciones juveniles hacen con ella y por cómo se la pone en relación con sus prácticas políticas y, específicamente, organizacionales.
} 
Las técnicas de recogida de datos fueron: a) encuesta, como modo de aproximación que nos permitió construir el primer mapa de situación; b) entrevistas individuales en profundidad; c) entrevistas colectivas semiestructuradas; d) observación no participante en relación con las instancias deliberativas y resolutivas que se generen entre los jóvenes; e) creación de espacios virtuales de intercambio entre jóvenes de Viedma y Mexicali.

La incorporación de técnicas diversas obedeció al propósito de mejorar la comprensión acerca de los ejes y dimensiones del esquema de análisis, explorar otros que fueron surgiendo en el proceso y realizar una tarea de triangulación y validación de resultados.

\section{Experiencias de politización juvenil en Viedma}

En el presente siglo se produjeron diversas experiencias de politización ${ }^{9}$ juvenil en Viedma que parecen, en algunos casos, desbordar la política institucionalizada, aunque no necesariamente, los jóvenes, dejan de ser interpelados por ella.

En una ciudad predominantemente administrativa como lo es Viedma ${ }^{10}$, capital de la provincia de Río Negro y sede del poder político provincial, un conjunto importante de instituciones estatales, organizaciones, partidos políticos, medios de comunicación y agentes especializados interactúa cotidianamente con la población joven de la ciudad,

\footnotetext{
${ }^{9}$ Compartimos la perspectiva que plantean Bonvillani, Palermo, Vázquez y Vommaro (2010) sobre los conceptos de politicidad y politización. Los investigadores sostienen que la politicidad de las prácticas no es algo evidente, sino que, por el contrario, merece ser desentrañada aun cuando todo tipo de lazo sea susceptible de politizarse. La politización es un potencial u horizonte constitutivo de cualquier vínculo social; sin embargo, es necesario ya que los autores plantean la necesidad de reconocer algunos aspectos que les permiten reconocer el carácter político de un colectivo o sistema de prácticas sociales. "La posibilidad de reconocer el carácter político de un conjunto de prácticas no convencionales o no institucionales, no significa considerar que todas las prácticas juveniles sean políticas per se. En estos términos sería muy difícil aprehender las características que le imprimen este carácter específico a las prácticas juveniles. Reconocemos que la politización es un potencial u horizonte constitutivo de cualquier vínculo social. Sin embargo, para atribuirle carácter político a un colectivo y a un sistema de prácticas sociales consideramos que es preciso reconocer, al menos, cuatro aspectos: 1) que se produzca a partir de la organización colectiva; 2) que tenga un grado de visibilidad pública (ya sea de un sujeto, de una acción o de una demanda); 3) que reconozca un antagonista a partir del cual la organización adquiere el potencial político; 4) que se formule una demanda o reclamo que, por lo dicho, adquiera un carácter público y contencioso" (Bonvillani y otros, 2010: 27-28).

${ }^{10}$ Viedma es la capital de la provincia argentina de Río Negro. Está situada en el noreste de la Patagonia, a 970 kilómetros la ciudad de Buenos Aires, sobre la ribera sur del río Negro, frente a la localidad de Carmen de Patagones. El río sirve de límite entre la provincia de Buenos Aires, a la que pertenece Carmen de Patagones, y la provincia de Río Negro. El Estado es la principal fuente de recursos y empleo en la ciudad. La principal actividad es la administración pública, si bien a partir de la década del noventa su participación en el empleo ha disminuido, sigue siendo dominante: un tercio (32\%) de los ocupados trabaja para el Estado (administración provincial, administración municipal, educación y salud pública), pero la dependencia del Estado se comprende mejor si se tiene en cuenta que el comercio, los servicios y la industria funcionan en relación directa con la evolución del gasto público. Solo la actividad ganadera o agropecuaria que logra colocar su producción fuera del mercado local puede considerarse una actividad con cierto grado de autonomía; sin embargo, también recibe aportes del Estado a través de subsidios, créditos, exenciones impositivas o por la construcción de obra pública.
} 
crea categorías sociales en función de las cuales se clasifican y construye a los grupos sociales, y despliega diversos y contradictorios discursos interpeladores de lo juvenil. Las identificaciones políticas juveniles se configuran en el marco de estas interpelaciones.

Las experiencias de politización juvenil estudiadas dan cuenta de la diversidad y complejidad de los procesos de identificación política ${ }^{11}$.

Las prácticas y modos de vivenciar la política de la mayoría de los jóvenes viedmenses están atravesados, entre otros aspectos, por su experiencia de habitar en los barrios pobres de la periferia de la ciudad.

La interpelación familiar aparece como una de las principales motivaciones entre los que se inclinan a militar en partidos políticos o en proyectos sociales de largo plazo. Pero muchas experiencias de politización juvenil surgieron en microespacios de participación y solidaridad, interpelados por referencias y referentes transitorios con los cuales encuentran una empatía, una identificación. Entre esos referentes transitorios, muchos de ellos surgieron y/o alcanzaron visibilidad a través de los medios de comunicación y, crecientemente, a través de las redes sociales digitales.

Los intereses y motivaciones giran en torno a cuestiones vinculadas con el reconocimiento de las diferencias, la reafirmación identitaria (pueblos originarios, culturas de algunos países limítrofes, como Chile o Bolivia), el medio ambiente o el reclamo por justicia, vivienda y educación. Estos polos de identificación se configuran y reconfiguran permanentemente en espacios que permiten su articulación política.

La política además de ser reconocida como una actividad vinculada con los partidos y el Estado, se ha desplazado a otros lugares y se ha vinculado con otros sujetos e incluye el orden de lo cotidiano. La dimensión personal y performativa de la política que se construye en las redes sociales digitales también permea las prácticas políticas juveniles en los espacios más tradicionales (partidos políticos, sindicatos, agrupaciones universitarias, entre otros) (Poliszuk, 2013).

\footnotetext{
${ }^{11}$ Una investigación sobre agregaciones juveniles en Viedma que llevamos a cabo entre 2007 y 2010, titulada Producción de sentidos en jóvenes de Viedma Patagones y nuevas formas de subjetivación política (Directora: Mg. Sandra Poliszuk/Co-directora: Mg. Raquel Borobia, UNComahue/PRIN 04/V054), nos permitió organizar de la siguiente forma los grupos juveniles que relevamos en el campo: 1: experiencias colectivas vinculadas con la ocupación de tierras; 2: grupos relacionados con prácticas artísticas; 3: grupos afines con movimientos mapuches; 4: grupos conexos a la reivindicación de derechos humanos; 5 : grupos de estudiantes; 6: grupos comprendidos en partidos políticos; y 7: grupos religiosos. A partir de la identificación de cuatro subgrupos constitutivos de la población, comenzamos la selección de los casos. Como resultado de la selección, trabajamos con doce casos a partir de los cuales pudimos construir cuatro tipos de agregación. Los cuatro tipos de agregación fueron caracterizados de la siguiente manera: Primer tipo: "grupos numerosos que forman parte de organizaciones mayores"; segundo tipo: "grupos numerosos que actúan en instituciones"; tercer tipo: "grupos de variada extensión que actúan por fuera de las instituciones, pero en relación con ellas"; cuarto tipo: "pequeños grupos independientes" (Poliszuk y Borobia, 2010).

En el presente trabajo se recuperan dos casos del segundo tipo.
} 
Entre las agregaciones estudiantiles estudiadas evidenciamos una creciente tendencia a desdibujar los límites de acción entre un adentro y un afuera de las instituciones educativas. En general, estos colectivos buscan trascender las demandas puramente escolares o universitarias, actuar tanto dentro como fuera de las instituciones estatales y discutir problemáticas sociales diversas, muchas de las cuales trascienden el ámbito educativo.

La participación política juvenil, en varios de estos casos, está ligada tanto a la acción directa en los espacios públicos, para canalizar algunos conflictos, y la horizontalidad, para la toma de decisiones de algunos asuntos, como así también a la delegación o representación en los ámbitos formales por situaciones que así lo requieran.

Asimismo, la política es vivida crecientemente por muchos de estos jóvenes en torno a identificaciones políticas provisorias más que a la construcción de alteridades relativamente estables. Esta característica se expresa, entre otros aspectos, en los modos de autonombrarse como grupo, ya sea fusionando conceptos, aparentemente contradictorios entre sí, que ponen en tensión sus sentidos hegemónicos o los subvierten, como así también apelando a nombres de la vida cotidiana o mezclando lenguajes que desestabilizan e incomodan los modos tradicionales de reconocimiento político.

En ese entramado, las redes sociales digitales constituyen, más que un simple canal de expresión, un escenario configurativo de las prácticas políticas, las formas organizativas y los procesos complejos de la construcción identitaria juvenil, donde se disputan los sentidos y se resignifican las diferentes trayectorias grupales.

\section{Sentirse y vivenciar el espacio tiempo y la intimidad en las redes sociales digitales: el caso de una agrupación estudiantil universitaria}

En este apartado se presenta, en primer lugar, la trayectoria de una agrupación estudiantil universitaria de Viedma. Luego, a partir de las entrevistas realizadas a sus integrantes, se identifican, en sus prácticas comunicativas, los modos de sentir(se) en las redes sociales digitales, con el fin de analizar el impacto de esas sensibilidades en las interacciones, los lenguajes y las formas de organizarse, en general, de la agrupación. Partimos de entender que los modos de sentir y vivenciar el espacio tiempo y la privacidad en las redes sociales digitales impactan en las experiencias de militancia y las reconfiguran.

Las sensibilidades, como dimensión constitutiva de las prácticas sociales, resignifican las dinámicas de relación en las redes sociales digitales y van dejando sus marcas en las maneras de sentir y vivir la militancia universitaria.

El análisis se detiene en las sensibilidades de lo inmediato, lo instantáneo, lo simultáneo y lo cercano -producidas en las redes sociales digitales-, y la relación que mantienen con formas de vivenciar la comunicación en otros espacios de militancia y en otros momentos de la trayectoria de la agrupación. En ese sentido, los jóvenes 
activan sentires anteriores frente a las nuevas formas de expresión y sensibilidades y estas, a su vez, son incorporadas -no sin conflictos, ambigüedades, ambivalencias- a los modos de organización y comunicación de la agrupación, reconfigurándolos.

En relación con su trayectoria, la agrupación estudiantil universitaria cuenta con más de 25 años de vida. Surgió a principios de la década del 90 y durante varios períodos condujo el Centro de estudiantes de la sede de la Universidad del Comahue, en Viedma. Su singularidad radica, entre otros aspectos, en los desplazamientos de su construcción identitaria y en sus formas organizativas. La agrupación estuvo conformada, en sus inicios, por estudiantes militantes con afiliación partidaria y estudiantes denominados "independientes". Su identidad comenzó a configurarse a partir de dos significantes clave que aunaron y tensionaron esa construcción: "movilización" e "independencia". Entre 1995 y 2000, la agrupación formó parte de la fuerza del socialismo en el ámbito universitario. A partir del nuevo siglo, comenzó un proceso interno de cuestionamiento a la pertenencia partidaria, al verticalismo en las estructuras formales, y se impulsó la reorganización y búsqueda de autonomía de la agrupación. En 2004, la agrupación decidió no formar parte de la estructura del socialismo, se sumó a una federación de organizaciones estudiantiles universitarias independientes y se proyectó hacia afuera del ámbito universitario, en alianza con algunos movimientos sociales de la región. En 2005, la agrupación lideró una "toma" estudiantil de la sede universitaria en Viedma por la democratización del gobierno universitario, en continuidad con otras "tomas" estudiantiles producidas en diversas sedes de la misma universidad ${ }^{12}$, en 2004 , en rechazo a la CoNEAU y a la Ley de Educación Superior. Este acontecimiento constituyó un momento clave en la trayectoria de la agrupación, fue una modalidad de irrupción novedosa, aunque cuestionada por las autoridades universitarias $y$, posteriormente, por una gran parte del estudiantado. La toma significó un espacio para la emergencia de nuevas formas de expresión grupal, nuevas sensibilidades y modos de habitar el espacio. La propuesta de mantener "abierta" la universidad para la realización de diversas actividades con las organizaciones sociales de la ciudad les permitió profundizar en la reorientación identitaria de la agrupación. La convivencia permanente de los estudiantes, con otros actores sociales de la comunidad, en tanto modalidad predominante de apropiación del tiempo-espacio de la toma, fue central en la reafirmación de la identidad grupal; especialmente, en la dimensión afectiva, pasional, de la experiencia. Pero, en un escenario de creciente tensión entre demandas diferentes y lo que el orden

\footnotetext{
${ }^{12}$ La Universidad Nacional del Comahue está asentada en un territorio amplio que abarca tres provincias argentinas: Neuquén, Río Negro y Chubut. Su sede central está ubicada en la ciudad de Neuquén y tiene dependencias en Viedma, Bariloche, San Martín de los Andes, Cipolletti, Zapala, Allen, General Roca, Cinco Saltos, Choele Choel, San Antonio Oeste, Villa Regina, Esquel, Puerto Madryn y Trelew. La Universidad cuenta con 12 Facultades y sus 2 Asentamientos Universitarios dependientes, una Escuela Superior y 2 Centros Regionales.
} 
institucional pudo absorber, la posición de la agrupación perdió representación dentro del sector estudiantil, poniéndose en juego la exigencia de normalización del dictado de clases.

Uso de las redes sociales digitales: para analizar los usos de las redes sociales digitales de la agrupación, partimos de comprender, junto con Rosalía Winocur (2012) que la incorporación de un nuevo medio de comunicación siempre ha estado mediado por experiencias anteriores $y$, también, por los imaginarios sociales que establecen funciones, sentidos y prescripciones, desde los cuales son apropiados los nuevos medios. Los usos de las redes sociales digitales están mediados por representaciones sociales que operan aún antes que los sujetos tengan acceso al nuevo medio. Desde esta perspectiva, para conocer cuáles son sus usos y cuál es el significado de esa experiencia mediática para quienes los utilizan, se requiere tener en cuenta, en primer lugar, los contextos constitutivos específicos de sus prácticas comunicativas.

Si bien el perfil en Facebook de esta agrupación fue creado en 2009 y comenzó a ser usado para conectarse con otras agrupaciones estudiantiles independientes que intervenían en distintas sedes universitarias de la región -alejadas muchas de ellas por más de $600 \mathrm{~km}$-, las redes sociales digitales se insertan dentro de un conjunto de otros medios, espacios de interacción y saberes que forman parte de sus prácticas comunicacionales. En ese sentido, la agrupación ha venido utilizando desde este nuevo siglo las diversas plataformas de Internet, las cuales se fueron integrando a los espacios presenciales de interacción, difusión y debate marcados, predominantemente, por la exposición argumentativa de sus ideas. En 2008, algunos integrantes de la agrupación generaron un proyecto comunicacional que consistió en un blog de debate político y social frente a lo que consideraban una necesidad de expresarse y participar por fuera de los ámbitos institucionalizados por falta de espacios para hacerlo. Estos jóvenes visualizaban, en ese momento, que Internet ampliaba el intercambio y el debate con otros actores locales y regionales. Durante el año 2009 se registró la mayor intensidad de publicaciones en el blog, con entrevistas a referentes académicos e intelectuales del ámbito político regional, notas de análisis socio-político regional y local, y comentarios y reflexiones sobre la política universitaria.

Las redes sociales digitales como Facebook fueron usadas, en un principio, como una vía excepcional en un momento de crisis de la Federación, en el cual algunas agrupaciones tendieron a desaparecer. Facebook se utilizó, en un primer momento, únicamente como grupo cerrado que permitía saber sobre "el día a día" de las agrupaciones que compartían la Federación. Esta posibilidad de interacción en forma continua fue valorada positivamente desde sus comienzos. Lo que adquirió mayor valor fue lo que conectaba a estos jóvenes con el presente de la Federación, con lo que estaba pasando en sus redes de contacto, lo que a su vez hizo que lo instantáneo adquiriera importancia. La comunicación por la red digital Facebook les permitió 
sentirse parte de ese presente continuo, una experiencia del tiempo vivido estructurada con la sensibilidad de lo inmediato y lo instantáneo.

Esta modalidad de uso, únicamente como grupo cerrado, se utilizó para momentos y circunstancias particulares -especialmente, para comunicarse con otras agrupaciones de la Federación- y estuvo delimitado temporalmente -solamente cuando se hacía imposible que un representante de la agrupación viajara a otra ciudad para los encuentros presenciales de la mencionada Federación-. Cuando no era posible este contacto cara a cara, Facebook estaba disponible para recrear algunos códigos de la presencialidad, promoviendo una sensación de cercanía o proximidad con otras agrupaciones. La comunicación por grupo cerrado buscaba dar continuidad e imitar, hasta cierto punto, la comunicación presencial, pero no la sustituía, porque los integrantes encontraban limitaciones para debatir y tomar decisiones colectivas por este medio.

A partir de 2010, se le agrega crecientemente la modalidad de uso público de Facebook para conectarse con el estudiantado, en general, con docentes, espacios institucionales de la universidad y organizaciones sociales de la región. Este cambio en la modalidad de uso coincidió con un momento de recambio al interior de la agrupación cuando el grupo más activo, en ese momento, dejó la universidad y un sector de los "allegados" se conformó como nuevo grupo activo. El uso público de Facebook se intensificó en 2011. Los modos de organización de la agrupación también fueron cambiando en ese período al que los propios entrevistados lo autodenominan como un "nuevo período" más centrado en "la horizontalidad". El grupo de estudiantes activos se redujo, y se intensificaron la rotación y los tiempos alternados de militancia. La intensidad vivida en la etapa de la toma y los sentires de una convivencia compartida en un espacio único y una temporalidad permanente no parece caracterizar a este período, marcado por la temporalidad alternada como modalidad predominante de vivenciar la militancia.

La incorporación y uso público de Facebook es percibido por los entrevistados como una "demanda de la masa estudiantil" y como un medio más para la comunicación grupal; no es el único, aclaran, aunque lo perciben como importante y conveniente para llegar a diversos grupos y espacios institucionales.

El uso creciente de Facebook es proporcionalmente inverso al uso del blog: si bien ambos medios fueron utilizados con intensidad durante el período 2009-2012, el blog declinó su actividad a partir de 2012 y el uso de Facebook se incrementó desde ese año hasta la actualidad.

Se fue dimensionando la finalidad de esta red social: "Ilegar más" a los estudiantes con informaciones u opiniones, y vincularse con otras organizaciones sociales de la región y con un público más amplio. Los integrantes de la agrupación fueron adquiriendo mayores habilidades y predisposición para usar Facebook, y se comenzó a utilizar con más frecuencia con el fin de convocar a actividades y manifestaciones, 
resaltar los logros del grupo, señalar quiénes son sus adversarios o sostener algunas causas sociales particulares. La actividad en el perfil aumentó en los últimos años: se comenzó a subir diariamente alguna información y "si un día no hay información (sostiene una entrevistada), se sube una frase o una reseña para pensar". El repertorio de mensajes se diversificó, se entremezclaron los lenguajes de las fotografías, los emoticones y las palabras. Se intensificó la producción de relatos que combinan lo lúdico y lo reflexivo, lo grupal y lo individual, lo público y lo privado.

Mientras que el perfil en Facebook tiene un predominio de micropublicaciones, el blog estaba centrado en publicaciones extensas de análisis político.

Por otra parte, los integrantes de la agrupación fueron percibiendo que las convocatorias a través de esta red social son más rápidas, menos trabajosas. La inmediatez se enmarca en la lógica de la velocidad que rige a las tecnologías informáticas estructurando la experiencia del tiempo vivido con la sensibilidad de lo inmediato y lo instantáneo, imprimiéndole a la vida del grupo su propia huella.

Sin embargo, en la medida en que se fue intensificando la apropiación grupal de este nuevo medio, también surgieron problemas, se tomaron distancias del dispositivo y en la actualidad la agrupación cuestiona algunas de sus propias prácticas comunicativas.

Por un lado, sienten que Facebook parece acercar a los estudiantes que están lejos, en otras sedes universitarias de la región, pero aleja a los que están cerca, en la propia sede universitaria. Esta particularidad se evidenció en el fracaso de las convocatorias a través de esta red cuando hay que involucrar el cuerpo en la acción directa.

Otro aspecto que se presentó como problemático tiene que ver con la intimidad que se muestra en las redes. Las discusiones al interior del grupo y el modo en que se toman las decisiones llevaron a estos jóvenes a delimitar los aspectos íntimos que, consideran, deben quedar ocultos, de aquellos otros que pueden ser mostrados en el ciberespacio. La creación de perfiles paralelos es una práctica habitual que exige un esfuerzo permanente por delimitar la intimidad. Así, la delimitación de la intimidad/publicidad del grupo en las redes no surge espontáneamente, sino que implica un trabajoso esfuerzo del sujeto para demarcar sus límites y requiere invertir una cantidad considerable de energías.

\section{Facebook: otro lugar entre la escuela y la radio. El caso de un grupo de estudiantes secundarios nucleados en torno a la comunicación popular}

En este apartado se analizan las prácticas comunicacionales de un grupo de estudiantes de una escuela de Nivel Medio, de formación agraria, ubicada en la zona semi rural, a $30 \mathrm{~km}$ de Viedma. Este grupo se nuclea, principalmente, en torno a un proyecto de radio escolar comunitaria que funciona desde hace 7 años en la mencionada institución educativa. Está conformado, en su amplia mayoría, por estudiantes que viven en Viedma y viajan diariamente hasta dicha escuela. El proyecto de la radio se viene desarrollando con la coordinación de un trabajador social, 
integrante del área no formal de la escuela, con el apoyo del equipo de comunicadores de una radio comunitaria viedmense que funciona desde 1992 y tiene una fuerte presencia en el campo de la comunicación popular latinoamericana.

La propuesta de la radio escolar comunitaria surgió a partir de una serie de talleres sobre derechos humanos e identidades que organizó el área no formal de la escuela. Los objetivos iniciales que se plantearon los estudiantes en el proyecto fueron: "conectar a la escuela con la comunidad y revertir la imagen negativa que tienen los jóvenes en la comunidad".

El proyecto tomó impulso y fue posible la instalación de la radio a partir de la participación de los estudiantes en un concurso organizado por el Programa Nacional para Adolescentes Nuestro Lugar, del Ministerio de Desarrollo Social de la Nación, del cual resultaron ganadores de un premio que consistió en el aporte económico para la compra de parte del equipamiento de la radio. En el año 2012 obtuvieron la licencia y en 2013, la radio comenzó a funcionar.

De esta manera, el interés de estos jóvenes por la radio los ha llevado a realizar radios abiertas en distintos eventos sociales, a coordinar actividades con diversas organizaciones estableciendo vínculos intergeneracionales e intersectoriales. También vienen participando en capacitaciones sobre radiodifusión brindadas por docentes de la carrera de Comunicación Social de la Universidad Nacional de Río Negro, por la Fundación Alternativa Popular (Radio Encuentro) y con el apoyo de la Subsecretaría de Agricultura Familiar de la Nación y organismos no gubernamentales.

El grupo creador e impulsor del proyecto se constituyó a partir de 20 estudiantes aproximadamente. Luego se fueron sumando otros jóvenes de la misma escuela. La cantidad de integrantes fluctúa año tras año, pero se mantiene entre 50 y 70 estudiantes. Muchos de estos jóvenes también participan en otros colectivos, agrupaciones y en el centro de estudiantes de la escuela. La pertenencia simultánea a varios tipos de agregaciones, con objetivos diversos, los lleva a preferir el funcionamiento en redes como modalidad de trabajo.

La experiencia de la radio escolar comunitaria se constituyó para estos jóvenes en un espacio para reflexionar sobre la comunicación como un derecho y un ámbito que promueve la recuperación de las culturas populares, regionales y locales, la participación y la toma de posición frente a temas de interés común, como así también el compromiso con los sectores sociales más empobrecidos y excluidos.

El proyecto de la radio les posibilita a estos jóvenes tomar distancia de los lugares asignados en el ordenamiento jerárquico de la institución y entablar relaciones intergeneracionales de mayor reciprocidad. La puesta en marcha de la radio implicó poner en tensión el ordenamiento espacio temporal de la escuela, entre otros dispositivos reguladores de subjetividad, movimientos que no estuvieron ni están exentos de luchas y conflictos. Por un lado, la radio impulsó otros modos de 
organización del trabajo escolar, de interacción al interior de la escuela y con la comunidad.

En este contexto, lo que se puso en disputa fue el lugar de alumno que se les tiene asignado a los jóvenes dentro de la jerarquía institucional. Ellos fueron redefiniendo su posicionamiento en la escuela, operación que implicó recusar gradualmente ese lugar.

Otro componente en tensión fue el ordenamiento espacio temporal: los jóvenes conviven con otras temporalidades y con otros modos de habitar los espacios que los que impone la escuela. En ese sentido, como ya se desarrolló anteriormente, las redes sociales digitales generan y promueven una particular manera de sentir el tiempoespacio, ligado a la cercanía y a la instantaneidad.

En cuanto a la participación, los estudiantes han promovido actividades que trascienden los objetivos de la institución y se proyectan hacia la comunidad. Muchas de estas actividades se organizan a través de las redes sociales digitales, y muchas de ellas conservan la lógica de agruparse y reagruparse a partir de referencias transitorias y reclamos puntuales. Sin embargo, también se perciben tensiones y conflictos en este sentido, cuando se intenta imponer, desde una mirada adultocéntrica, parámetros de participación teñidos de nostalgia de otras épocas.

En ese sentido, las redes sociales digitales se convirtieron en otro lugar de resistencia de los estudiantes a los intentos de controlar institucionalmente el funcionamiento de la radio. Un modo de experimentar el poder de las comunidades virtuales fue a través de la vivencia de "autonomía" frente a los adultos -padres, autoridades educativas o docentes- que produjo en los jóvenes un estado de sentir asociado a la conquista de un lugar propio. Para estos jóvenes, el uso de un perfil en Facebook de la radio connota la posibilidad de una esfera de lo propio con mayor autonomía de los adultos y que da lugar a relaciones con otras personas.

En las redes sociales digitales llevan a cabo una intensa actividad. Desde Facebook se sienten acompañados por los oyentes y el perfil de la radio se usa, además, como mediador cuando hay conflictos en el grupo y se bloquean los perfiles individuales. Desde la mirada de algunos de ellos, Facebook es lo que le da vida a la radio. En una zona semi rural y con una conectividad dificultosa, las redes digitales los mantienen conectados con los oyentes $y$, especialmente, con otros jóvenes que viven en Viedma, donde no llega la señal de la radio por su corto alcance.

El uso de Facebook surgió, en un primer momento, como un medio complementario de apoyo al proyecto, con el fin de que este tuviera una mayor repercusión. Pero pronto las redes sociales digitales pasaron a ocupar otro lugar y tener otras funciones. Una estudiante entrevistada señala:

Más allá de que nos escuchen, queríamos ocupar otro lugar, y usarlo como medio de comunicación al Face, pero tener otro lugar, porque hay otras personas en el Face, hay otras edades en el Face, y no es la misma gente la que nos escucha a las 9 de la mañana y la que nos pone "me gusta" a las 6 de la tarde (E4). 
La utilización de Facebook aparece como una práctica que, en parte, se resiste a la institucionalización de la radio y a la cultura escolar dominante. Los jóvenes valoran el sentirse en conexión de igual a igual con otros jóvenes y recuperan algunos valores y sentires vividos en la experiencia inicial de funcionamiento de la radio, inscripta en la tradición residual de la comunicación popular. La sensación de horizontalidad es uno de esos sentires que estructura la trayectoria del grupo y que se resignifica con el uso de las redes digitales. La necesidad de vivenciar la horizontalidad estructura la experiencia y la aspiración de estos jóvenes; pero la cultura institucional dominante, con renovados mecanismos, minusvalora, contradice e incluso es incapaz de reconocer.

Esta experiencia demuestra que los elementos de resistencia, oposición o alternativos, del proceso, constantemente se renuevan, como así también los mecanismos de la cultura dominante que buscan incorporar y adaptar los elementos genuinamente creativos de lo nuevo en una dinámica que nunca se agota.

\section{Conclusión}

Para concluir este trabajo, se recuperan las estructuras del sentir que fueron surgiendo en el análisis con el fin de proponer una lectura más amplia sobre sus impactos en el modo de vivenciar la militancia y en las prácticas comunicacionales en general -modos de expresarse, organizarse y participar- de las agregaciones juveniles estudiadas.

Los entrevistados han manifestado sentires como la inmediatez, la cercanía o proximidad, el temor a la falta de resguardo de la intimidad grupal y el creciente interés que les provoca el intercambio cotidiano en las redes sociales digitales. Estos sentires van naturalizándose y configurando un particular modo de vivir la militancia estudiantil en cada agregación estudiada. También se han reconocido formas específicas de pensar, sentir y actuar, configuradas como estructuras de sentir, en algunos conflictos vinculados a la experiencia del tiempo y el espacio, y la intimidad en las redes sociales digitales. Específicamente, el trabajo se ha centrado en las distancias y cercanías que promueven estas redes y que, en el proceso de apropiación grupal del primer caso presentado, fueron cuestionadas. Otro aspecto abordado como problemático fue el de la delimitación de la intimidad que se muestra en las redes y los modos de demarcarlos.

El trabajo permitió reconocer algunos modos en que las estructuras de sentir de las redes sociales digitales se integran o entran en tensión con sentires y prácticas comunicacionales previas a su uso.

Las estructuras del sentir, rastreadas en la conciencia práctica de las agrupaciones, indican formas específicas de pensamiento, sentimiento e interacción frente a las dificultades y los conflictos que surgen en la vivencia cotidiana de la militancia juvenil. El reconocimiento de las estructuras del sentimiento se vuelve significativo para 
comprender algunos procesos más amplios, configuradores del presente que forman parte del horizonte cultural de una época.

Para comprender el impacto de las estructuras del sentir en las experiencias de militancia estudiantil actuales, se requiere arriesgar una lectura del horizonte cultural actual en el cual se insertan. Las sensibilidades abordadas, en contextos de creciente mediatización, se palpan en un escenario cultural que habilita modos particulares de sentir-vivir la militancia estudiantil, asociada crecientemente a la multiplicidad de espacios de interacción y expresión, y temporalidades diversas que se entrecruzan y superponen en la vivencia cotidiana. Los sentires de cercanía e instantaneidad conviven con la temporalidad larga y las distancias jerárquicas propias de la espacialidad escolar y académica.

Los discursos motivadores, las narraciones fragmentadas y las micropublicaciones de Facebook se integran crecientemente al repertorio comunicativo de las agregaciones estudiadas $y$, junto a los modos particulares de sentir vivir el tiempo-espacio en las redes, van haciendo surgir una particular construcción de la militancia, ligada a espacios discontinuos, compromisos fragmentados y alternados, y a un relato interrumpido de la política.

Las prácticas comunicacionales juveniles en las redes sociales digitales se integran, no sin contradicciones y tensiones, a las trayectorias grupales y las reconfiguran. Las redes sociales digitales generan usos y producen sentires y apropiaciones muy variados, y hasta contrapuestos, según las agregaciones estudiadas. En las experiencias abordadas en este trabajo se evidenciaron modificaciones y resistencias a los cambios que conlleva la comunicación digital a través del registro de situaciones que, en un caso, suponen la apreciación de otras prácticas por encima de las nuevas $y$, en otro caso, mantienen la vigencia de algunos sentires residuales mientras se apropian de algunos elementos genuinamente creativos de lo nuevo.

Finalmente, este estudio nos lleva a plantear que recuperar la dimensión sensible de las prácticas comunicacionales permite reconocer más acabadamente las condiciones para ser reapropiadas y potenciadas en función de poder comprender y operar sobre los marcos de posibilidad y constricción de la acción colectiva.

\section{Bibliografía}

Arfuch, L. (2002). Identidades, sujetos y subjetividades. Buenos Aires: Prometeo Libros.

Arfuch, L. (2007). El Espacio Biográfico: Dilemas de la Subjetividad Contemporánea. Buenos Aires: Fondo de Cultura Económica.

Boito, M. E. (2010). Estados del sentir en contextos de mediatización y mercantilización de la experiencia. Intentos por precisar una lectura materialista de las sensibilidades. En Grosso, J. y Boito, M. E. Cuerpos y emociones en América Latina. Córdoba: CEA, CONICET. 
Balardini, S. (2006). Impacto y transformaciones de la cultura escolar ante la inclusión de las tecnologías de la información y la comunicación. Subjetividades Juveniles y Tecnocultura. Buenos Aires: FLACSO.

Bonvillani, A.; Palermo, Y.; Vázquez, M. y Vommaro, P. (2010). Del Cordobazo al kirchnerismo. Una lectura crítica acerca de los períodos, temáticas y perspectivas en los estudios sobre juventudes y participación política en la Argentina. En Alvarado, S. y Vommaro, P. (comps.) Jóvenes, cultura y política en América Latina: algunos trayectos de sus relaciones, experiencias y lecturas 1960-2000. Rosario: Homo Sapiens Ediciones, pp. 21-54.

Borobia, R. (2015). Estrategias metodológicas en estudios de subjetividades juveniles. En Mingo, G. y Sarrot, E. G. (comps.) Desafíos profesionales y prácticas académicas en el campo de la investigación y la producción metodológica. Ciudad Autónoma de Buenos Aires: Estudios Sociológicos Editora.

Cáceres Riquelme, J. y Herrera Pardo, H. (2014). Las formas fijas y sus márgenes: sobre "Estructuras de sentimiento" de Raymond Williams. Una trayectoria. Universum (Talca), 29, 1, pp. 173-191. Disponible en: https://dx.doi.org/10.4067/S0718$\underline{23762014000100010}$

Catelli, N. (2007). La Era de la intimidad. Buenos Aires: Beatriz Viterbo.

Hine, C. (2004). Etnografía Virtual. Barcelona: UOC.

López, G. y Ciuffoli, C. (2012). Facebook es el mensaje. Oralidad, escritura y después. Buenos Aires: La Crujía.

Martín Barbero, J. (1987). De los medios a las mediaciones. Comunicación, cultura y hegemonía. México: G. Gili.

Martín Barbero, J. (2002). Desencuentros de la socialidad y reencantamientos de la identidad. Revista Analisi, 29, pp. 45-62.

Martín Barbero, J. (1999). Los descentramientos del arte y la comunicación. En Ossa, C. (comp.) La Pantalla Delirante. Santiago de Chile: Lom Ediciones.

Néspolo, J. (2007). El problema de la identidad narrativa en la filosofía de Paul Ricoeur. Orbis Tertius, XII, 13. La Plata: Facultad de Humanidades y Ciencias de la Educación, UNLP, pp 1-14.

Poliszuk, S. y Borobia, R. (2010). Trayectorias juveniles y sentidos de la política. Revista Question, 1, 26. La Plata: Facultad de Periodismo y Comunicación Social, UNLP.

Poliszuk, S. (2013). La militancia de la vida propia en las redes sociales virtuales Representaciones juveniles de la identidad y de la política. En Ortiz Marín, M.; Poliszuk, S. y Barilá, M. I. (coords.). Las subjetividades juveniles en los entramados culturales contemporáneos. Un estudio con jóvenes argentinos y mexicanos. General Roca, Río Negro: Publifadecs.

Poliszuk, S. (2015). Ocupar otro lugar. Prácticas comunicacionales juveniles en las redes sociales digitales. Revista Argentina de Estudios de Juventud, 9. La Plata, Buenos Aires: Facultad de Periodismo y Comunicación Social, UNLP. 
Sibilia, P. (2008). La intimidad como espectáculo. Buenos Aires: Fondo de Cultura Económica.

Thompson, J. (1998). Los media y la modernidad. Barcelona: Paidós.

Verón, E. (1997). Esquema para el análisis de la mediatización. Revista Diá-logos de la Comunicación, 48, pp. 9-16.

Williams, R. (1997). Marxismo y literatura. Barcelona: Península.

Winocur, R. (2012). Transformaciones en el espacio público y privado. La intimidad de los jóvenes en las redes sociales. Revista Telos. Cuadernos de comunicación e innovación. Abril-Junio. Madrid: Fundación Telefónica. 